\title{
Characterize the Quantitative Dependence of 3D Image Quality on the Primary Inspection Parameters
}

\section{M3AR-14PN2301023 Technical Letter Report}

\section{September 2014}

AA Diaz

MR Larche

DL Baldwin

MS Prowant
TS Hartman

MK Edwards

CE Chamberlin 


\title{
DISCLAIMER
}

This report was prepared as an account of work sponsored by an agency of the United States Government. Neither the United States Government nor any agency thereof, nor Battelle Memorial Institute, nor any of their employees, makes any warranty, express or implied, or assumes any legal liability or responsibility for the accuracy, completeness, or usefulness of any information, apparatus, product, or process disclosed, or represents that its use would not infringe privately owned rights. Reference herein to any specific commercial product, process, or service by trade name, trademark, manufacturer, or otherwise does not necessarily constitute or imply its endorsement, recommendation, or favoring by the United States Government or any agency thereof, or Battelle Memorial Institute. The views and opinions of authors expressed herein do not necessarily state or reflect those of the United States Government or any agency thereof.

\author{
PACIFIC NORTHWEST NATIONAL LABORATORY \\ operated by \\ BATTELLE \\ for the \\ UNITED STATES DEPARTMENT OF ENERGY \\ under Contract DE-AC05-76RL01830
}

Printed in the United States of America

\author{
Available to DOE and DOE contractors from the \\ Office of Scientific and Technical Information, \\ P.O. Box 62, Oak Ridge, TN 37831-0062; \\ ph: (865) 576-8401 \\ fax: (865) 576-5728 \\ email: reports@adonis.osti.gov
}

\begin{abstract}
Available to the public from the National Technical Information Service, U.S. Department of Commerce, 5285 Port Royal Rd., Springfield, VA 22161 ph: (800) 553-6847 fax: (703) 605-6900

email: orders@ntis.fedworld.gov online ordering: http://www.ntis.gov/ordering.htm
\end{abstract}




\title{
Characterize the Quantitative Dependence of 3D Image Quality on the Primary Inspection Parameters
}

\section{M3AR-14PN2301023 Technical Letter Report}

\author{
AA Diaz \\ MR Larche \\ DL Baldwin \\ TS Hartman \\ MK Edwards \\ CE Chamberlin \\ MS Prowant
}

September 2014

Prepared for

the U.S. Department of Energy

under Contract DE-AC05-76RL01830

Pacific Northwest National Laboratory

Richland, Washington 99352 



\section{Acknowledgments}

The work reported here is a joint (collaborative) effort with Argonne National Laboratory (ANL), and was sponsored by the U.S. Department of Energy, Office of Nuclear Energy (NE), under U.S. Department of Energy Contract DE-AC05-76RL01830; Pacific Northwest National Laboratory (PNNL) Project No. 58745. Mr. Chris Grandy is the Technical Area Lead, and Mr. Bob Hill is the National Technical Director. Additionally, PNNL recognizes Mr. Tom Sowinski as the U.S. Department of Energy Technical Manager and Mr. Carl Sink as the HQ Program Director.

PNNL would like to thank Mr. Grandy for his guidance and technical direction throughout the course of this effort. The Technical Team would also like to thank our colleagues at ANL for working jointly with PNNL staff over the course of this collaborative effort.

At PNNL, the authors are indebted to Mr. Royce Mathews for providing in-Lab support across the entire spectrum of technical activities conducted on this project. The Team would also like to extend their gratitude to Ms. Lori Bisping for all of her hard work in providing administrative and financial reporting support to this project. Lori's attention to detail, expertise, and efficiency are second to none. Finally, the PNNL technical team would like to extend their thanks to Ms. Kay Hass for her ongoing support and technical editing expertise in preparing and finalizing this Technical Letter Report.

PNNL is operated by Battelle for the U.S. Department of Energy under Contract DE-AC0576RL01830. 



\section{Acronyms and Abbreviations}

$\begin{array}{ll}\text { ANL } & \text { Argonne National Laboratory } \\ \text { dB } & \text { decibel } \\ \text { ETU } & \text { engineering test unit } \\ \text { fiscal year } & \text { Material Testing Rig with Temperature Control (version 2) } \\ \text { MARICO-2 } & \text { megahertz } \\ \text { MHz } & \text { nondestructive examination (or nondestructive evaluation) } \\ \text { NDE } & \text { nickel } \\ \text { Ni } & \text { phased array } \\ \text { PA } & \text { phased-array ultrasonic testing } \\ \text { PA-UT } & \text { Pacific Northwest National Laboratory } \\ \text { PNNL } & \text { sodium-cooled fast reactor } \\ \text { SFR } & \text { signal-to-noise ratio } \\ \text { SNR } & \text { 22-element array prototype serial number 1 } \\ \text { SN1 } & \text { 22-element array prototype serial number 2 } \\ \text { SN2 } & \text { technical letter report } \\ \text { TLR } & \text { under-sodium viewing } \\ \text { USV } & \end{array}$





\section{Contents}

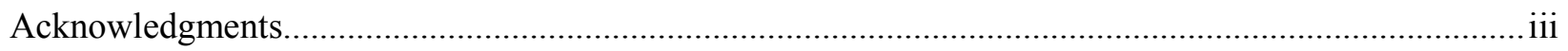

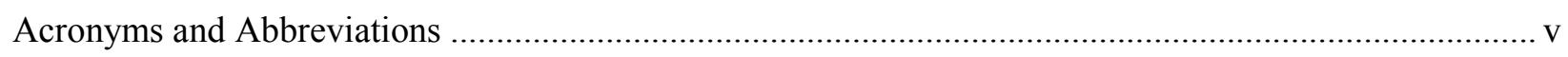

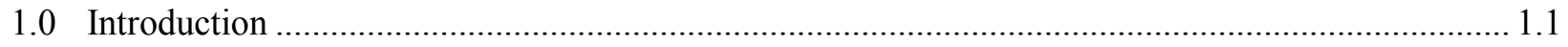

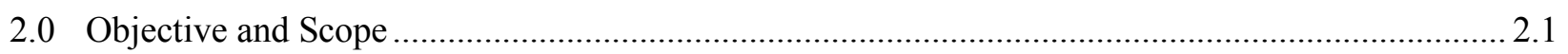

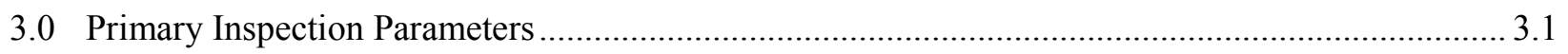

4.0 SN1 and SN2 Probe Design and Fabrication Differences ......................................................... 4.1

5.0 Imaging Assessment in Water for Prototype Probes .................................................................. 5.1

6.0 Imaging Assessment in Sodium for Prototype Probes.............................................................. 6.1

6.1 SN2 Probe Face and Sodium Preparations Prior to in-Sodium Scanning ................................ 6.1

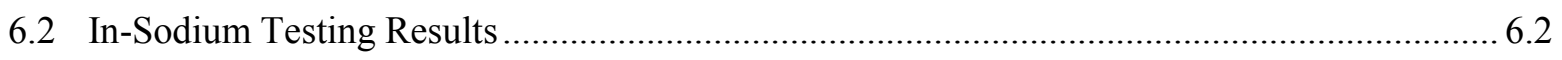

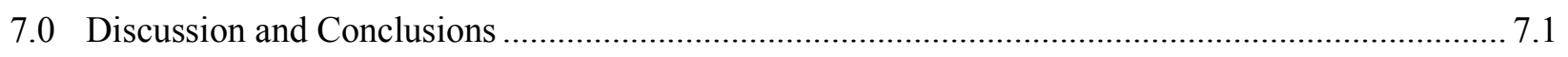

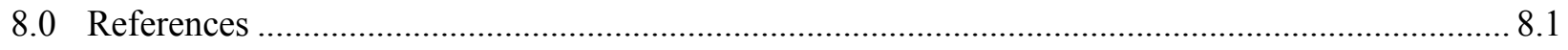




\section{Figures}

2.1 Side-view Schematic, Illustrating the Japanese Joyo Reactor Fuel Sub-assembly and the Associated Cross-sectional Dimensions of an Isolated Pin, within the MARICO-2 Test Sub-assembly

5.1 Three Individual Targets Employed for Water and In-sodium Imaging Tests .............................. 5.1

5.2 Top View of Target Position, Relative to Probe and Direction of Probe Rotation ........................ 5.2

5.3 Ultrasonic Images Resulting from Scans of Position 1, Target 1, at 50-mm Standoff Height in Water for the SN1 Prototype Probe

5.4 Ultrasonic Images Resulting from Scans of Position 1, Target 1, at 50-mm Standoff Height in Water for the SN2 Prototype Probe

6.1 Ultrasonic Images Resulting from Scans of Position 1, Target 1, at 50-mm Standoff Height in Sodium, at $260^{\circ} \mathrm{C}$, for the SN1 Prototype Probe.

6.2 Ultrasonic Images Resulting from Scans of Position 1, Target 1, at 50-mm Standoff Height in Sodium, at $260^{\circ} \mathrm{C}$, for the SN2 Prototype Probe. 


\subsection{Introduction}

This document is a Technical Letter Report (TLR) describing work conducted at the Pacific Northwest National Laboratory (PNNL) during FY 2014 on the under-sodium viewing (USV) project. This TLR satisfies the M3AR-14PN2301023 milestone, and is focused on characterizing the quantitative dependence of 3D image quality on the primary inspection parameters for in-sodium testing of the 22-element array prototype serial number 2 (SN2) prototype probe. This effort continues the iterative evolution supporting the longer term goal of producing and demonstrating a pre-manufacturing prototype ultrasonic probe that possesses the fundamental performance characteristics necessary to enable the development of a high-temperature sodium-cooled fast reactor (SFR) inspection system.

Sodium-cooled fast reactors are a technology of choice for advanced recycle reactors to be developed as part of the Generation IV Program. There is a need to re-establish the domestic technology infrastructure in order to support deployment of SFR technology. One key enabling technology is ultrasonic testing for under-sodium viewing that would be employed to (i) monitor operations in optically opaque sodium, and (ii) inspect structures, systems, and components within the reactor. PNNL's efforts are focused on demonstrating the use of linear phased-array ultrasonic probes to meet the needs of ranging and imaging in liquid SFRs. PNNL is currently developing linear phased-array ultrasonic testing (PA-UT) probes that are considered engineering test units (ETUs) that couple ultrasonic energy to the submerged structures of interest through liquid sodium. These probes provide the capability to image and conduct nondestructive examination (NDE) of critical components in high-temperature sodium-cooled fast reactors. The conceptual advantage of this approach is that the liquid sodium provides a medium that can directly couple the ultrasonic energy to the reactor components for imaging and inspection, if the liquid sodium is prepared, managed, and maintained appropriately (with regard to impurities and oxygen levels). The challenge is that the probe must withstand extended exposure to high temperatures and overcome wetting issues that can preclude the transmission of ultrasonic energy from coupling into the medium. PNNL demonstrated marginal success with the 22-element array prototype serial number 1 (SN1) PA-UT probe in FY 2013, but the signal-to-noise ratio (SNR) was poor for in-sodium testing trials.

Sodium-cooled fast reactors present some unique requirements in terms of technologies needed to support operations and maintenance. Inservice inspection and repair (ISI\&R) methods must be developed to support deployment of advanced SFRs. Such reactors will require high plant availability (capacity factor) and long lifetimes, and will require advanced ISI\&R technologies to ensure the integrity and safety of structures and components submerged in sodium, operating at elevated temperatures $\left(\sim 260^{\circ} \mathrm{C}\right)$. Key enabling technologies will allow operators to "see" through optically opaque sodium to support effective operations and maintenance activities. At the heart of the capability to image in sodium is the development of reliable probes to collect the basis data for reconstructing images of structures submerged in liquid sodium. This project is a joint Argonne National Laboratory (ANL) and PNNL project focused on developing, demonstrating, and optimizing probe platforms capable of supporting anticipated ISI\&R requirements. The baseline detection requirement, established during the first year (FY 2009) of the project, was derived from the need to detect a specific prototypic component (cross section of an isolated pin used within the MARICO-2 test subassembly). This benchmark has driven the prototype probe designs and performance evaluation methodologies. This report provides a summary of data, analyses, and the comparative results of water and in-sodium target detection tests using both the SN1 and SN2 prototype probes. 



\subsection{Objective and Scope}

An under-sodium viewing system will be an essential instrument for in-situ inspection of components of a sodium-cooled fast reactor. The USV system must be able to sustain effective operation in the hightemperature and corrosive environment of liquid sodium. At PNNL technical efforts are focusing on the development and demonstration of an effective and robust PA-UT imaging approach to address the inherent inservice inspection challenges associated with imaging and resolving the specified MARICO-2 pin cross section within the Joyo reactor fuel sub-assembly geometry.

The objective of the work conducted at PNNL and reported here is to demonstrate the ability to detect a target feature, equivalent to the largest cross section of an isolated pin used within the MARICO-2 test sub-assembly, submersed in $260^{\circ} \mathrm{C}$ liquid sodium. Figure 2.1 illustrates the Japanese Joyo reactor fuel sub-assembly and the cross sectional dimensions of a simulated pin. In the present fiscal year (FY 2014), emphasis is on completing the rebuild of the FY 2013 SN1 ETU PA-UT probe (with process enhancements), and testing this immersible linear array in water, hot oil $\left(260^{\circ} \mathrm{C}\right)$, and eventually sodium, at temperature.

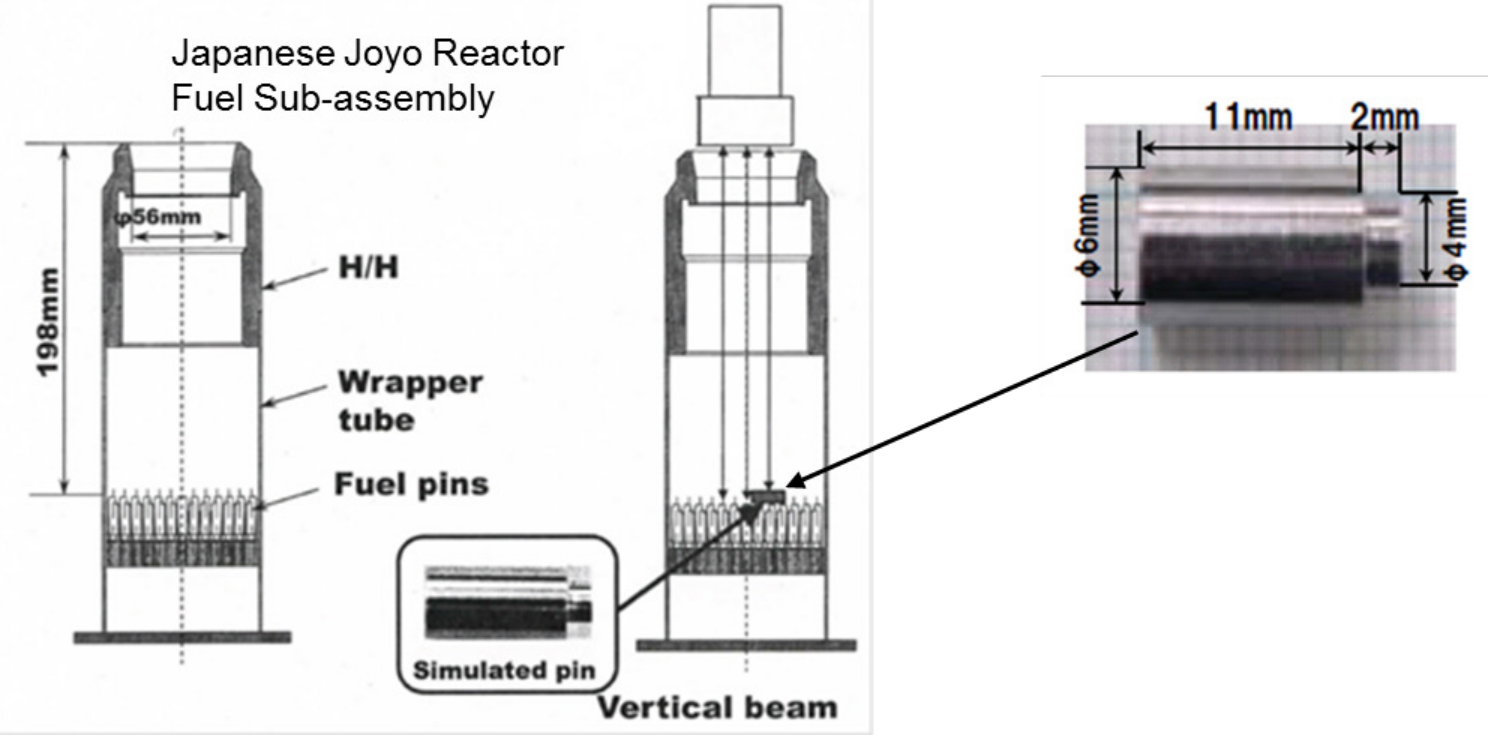

Figure 2.1. Side-view Schematic, Illustrating the Japanese Joyo Reactor Fuel Sub-assembly and the Associated Cross-sectional Dimensions of an Isolated Pin (simulated here), within the MARICO-2 Test Sub-assembly

The scope of the work for this portion of the PNNL effort conducted in FY 2014 includes documenting the outcomes of water and in-sodium testing, using both the SN1 and SN2 probes, and quantifying the dependence of the 3D image quality of these probes as a function of the primary inspection parameters. These inspection parameters include inspection time, spatial sampling frequency, sensor-to-target distance, sodium temperature, thermal cycling, etc. 



\subsection{Primary Inspection Parameters}

This section of the report defines the primary inspection parameters and critical attributes that provide the criteria for assessing the 3D image performance, functionality, and effectiveness of the SN1 and SN2 phased-array ultrasonic testing prototypes. The effort reported here is focused on analysis of data and performance metrics obtained from imaging work conducted with both prototype ETUs in water and sodium.

The primary inspection parameters and critical attributes for evaluating the $3 \mathrm{D}$ image quality of the SN2 probe in-sodium included:

1. Inspection time (duration)

2. Number of scanning repetitions

3. Data sampling frequency

4. Sensor-to-target distance

5. Spatial scanning increment size

6. Sodium temperature

7. Wetting of the probe face

8. Thermal cycling

9. Target size and orientation

10. Signal-to-noise ratio

11. Ability to resolve targets in-sodium

With the analyses of data obtained from these performance characterization tests, the PNNL Technical Team was able to quantify key performance parameters that were compared and contrasted between the two ETU probes, SN1 and SN2, for previous water and in-sodium tests in FY 2013 and FY 2014. Summary highlights of data and imaging results from water and in-sodium tests are provided in Sections 5.0 and 6.0, respectively, and the conclusions obtained from the evaluation of both probes are discussed in Section 7.0. 



\subsection{SN1 and SN2 Probe Design and Fabrication Differences}

This section of the report briefly summarizes the key design and fabrication aspects and differences between the SN1 and SN2 linear PA-UT probe designs, built at PNNL between FY 2013 and FY 2014, which to some degree, play a role in the differences between the imaging performances of the two probes. Prior to embarking on the design of the first-generation 22-element PA-UT prototype probe, PNNL fully documented the 9-element probe design and fabrication methods/processes. The Team then developed a test methodology, test targets, and a probe positioner for characterizing next-generation PA prototypes by $\mathrm{X}$-ray radiography, acoustic microscopy, and ultrasonic testing in water, hot oil, and in sodium. The 9-element probe was characterized and documented, and then the 22-element probe (SN1) was designed, fabricated, characterized, and tested in FY 2013. In FY 2014, the second-generation linear PA-UT probe (SN2) was developed. This design was similar to the design of the SN1 probe, but included modifications to the design and fabrication process protocols to accommodate lessons learned, improve SNR, and enhance overall probe performance.

As the project work unfolded in FY 2013, issues were identified relating to: (1) the bonding of the piezoelectric transducer multi-element crystal to the nickel (Ni) mounting substrate; (2) the laser machining of the phased-array element structures into the piezoelectric; (3) the reduced signal frequency of the 22-element phased array; (4) poor SNR; and (5) surface contour, thickness, and wetting issues (insodium). In FY 2014, the PNNL Technical Team focused on addressing these performance shortcomings with the SN1 probe by evaluating alternative methods, approaches, and processes associated with the design and fabrication stages for the SN2 probe. This included improvements and modifications to structural bonding processes, laser etching, soldering of the leads to the individual elements, ensuring a suitable bond of the multi-element crystal to the nickel substrate, improving surface polishing procedures, providing a thicker Ni faceplate, and enhancing sodium purification and regeneration processes to reduce impurities and oxygen levels in the sodium. The detailed description of the specific probe differences and performance variability between SN1 and SN2 prototypes has been previously documented (Diaz et al. 2014). 



\subsection{Imaging Assessment in Water for Prototype Probes}

This section describes the imaging tests and data obtained on the SN1 and SN2 prototype probes in water (at room temperature), to provide a baseline for target detection trials in-sodium. The scanning configuration for the water and in-sodium tests employed three different targets described previously (Braatz et al. 2013), and included six different scanning positions, representing a variety of target features as illustrated in Figure 5.1.

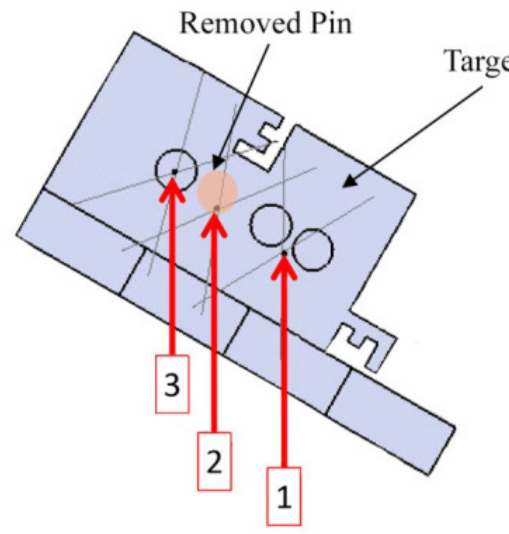

Target 1

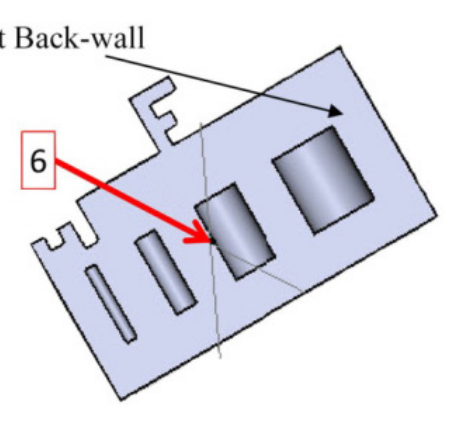

Target 3

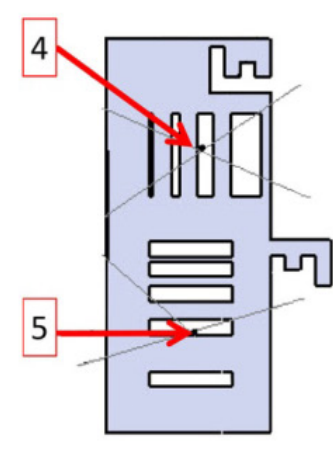

Target 2

Figure 5.1. Three Individual Targets Employed for Water and In-sodium Imaging Tests. Each numbered area on the targets represents an area where the probe was centered, for data acquisition.

At each position, when scanning a given target feature, the array was oriented directly over that position and subsequently rotated through $360^{\circ}$, in $0.5^{\circ}$ increments. Additionally, at each radial position, the sound field beam was then steered through angles of $-20^{\circ}$ through $+20^{\circ}$ at $0.5^{\circ}$ increment steps. Figure 5.2 illustrates this configuration over position 1, where a dual set of vertical pins are positioned.

During scanning, the probe was unable to acquire data at the designed focal depth of $76.2 \mathrm{~mm}$, due to a combination of physical constraints associated with the scanner mechanism (platform) and the dimensions of the sodium containment glove box. These same limitations were present during the data acquisition tests for the SN1 prototype probe, so both sets of tests were conducted using the exact same scanner and sodium containment. In FY 2013, the in-sodium SNR for imaging data using the SN1 probe was poor, and a special signal processing algorithm was developed in MATLAB to help discriminate relevant signal responses from noise and reconstruct an image of the targets in the sodium (Braatz et al. 2013). This tool was not used for image reconstruction of SN2 data, and the data from both the SN1 and SN2 probes provided here were compared and contrasted using Ultrasvision software, which is the commercial analysis tool that accompanies ZETEC phased-array ultrasonic imaging systems. 


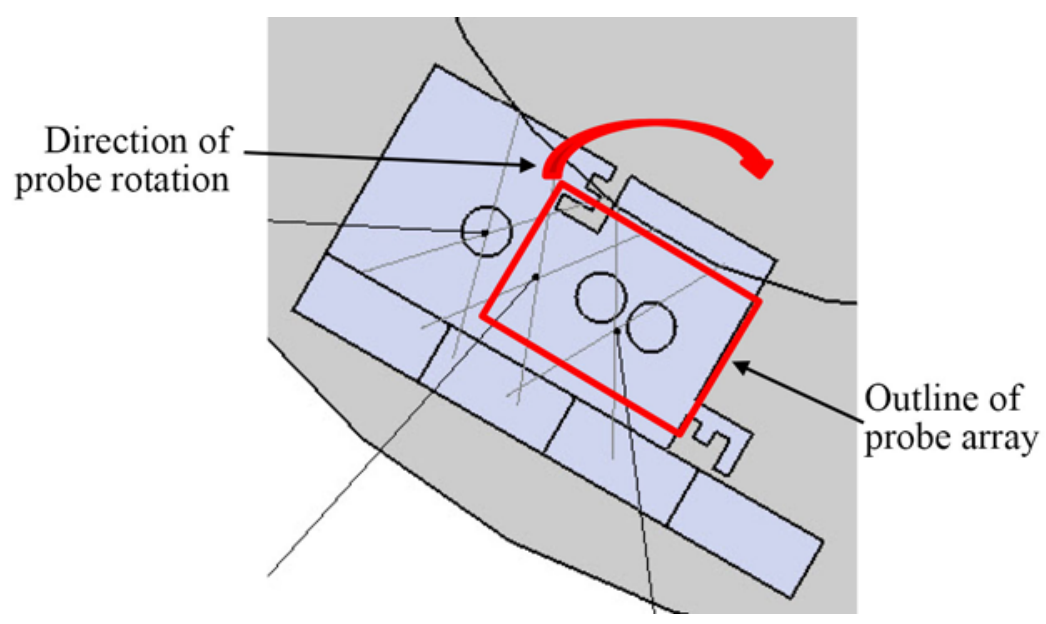

Figure 5.2. Top View of Target Position, Relative to Probe and Direction of Probe Rotation

In this section, we discuss the results from both prototype probes being scanned over position 1 of target 1 (dual pin set), which comprised two 6-mm diameter pins, spaced a distance of 1.23-mm apart. Each pin was $11 \mathrm{~mm}$ in height. Figure 5.3 illustrates the imaging results from data acquired using a $50-\mathrm{mm}$ standoff between the probe and the base of the target.

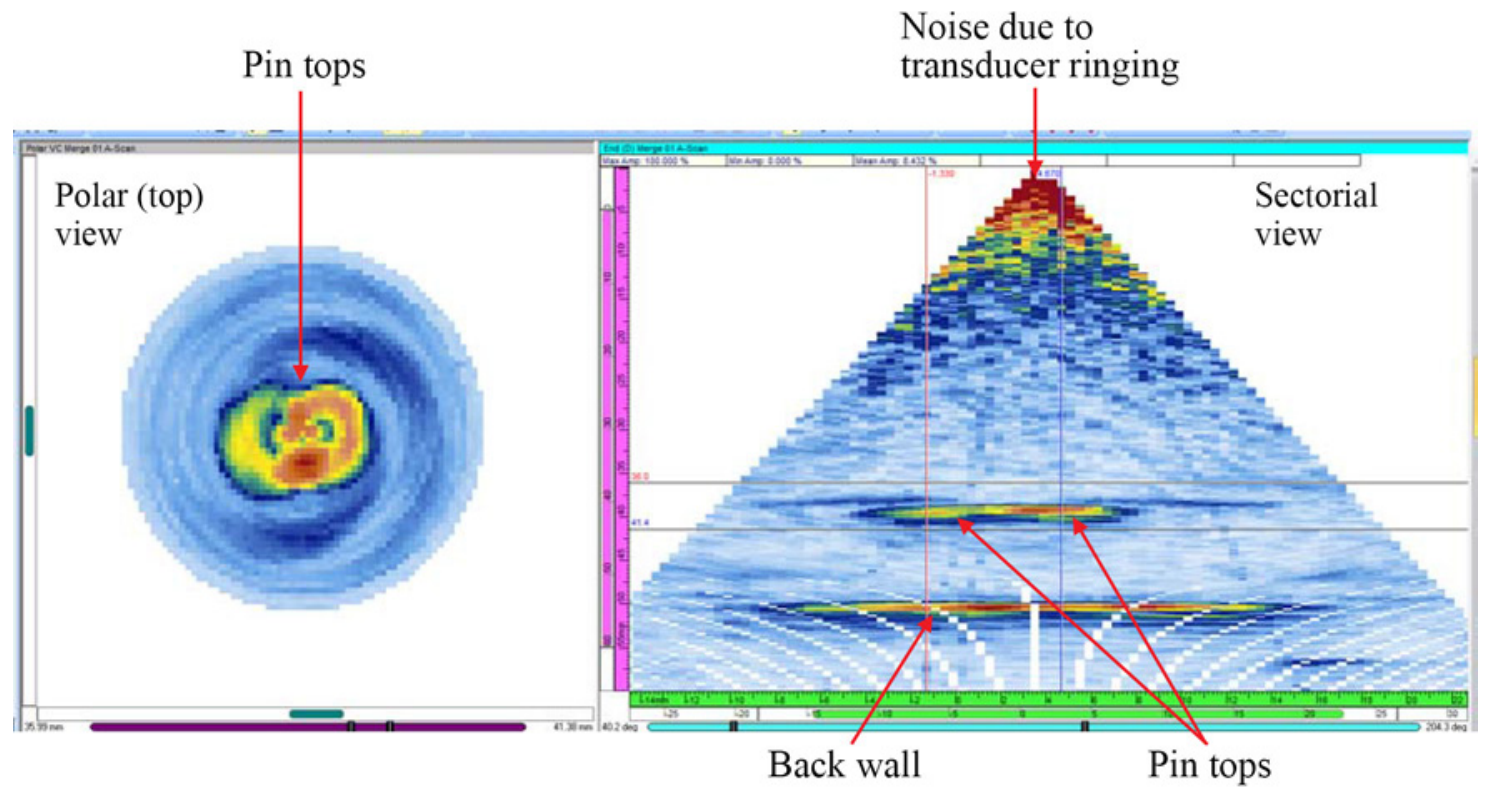

Figure 5.3. Ultrasonic Images Resulting from Scans of Position 1, Target 1, at 50-mm Standoff Height in Water for the SN1 Prototype Probe 
The tops of both pins can be distinguished from the back wall response in water, and the separation between the pins was measured to be $6 \mathrm{~mm}$. The height of the pins was measured as $11.1 \mathrm{~mm}$. The SNR was $20 \mathrm{~dB}$, which corresponds to a factor of 10:1 in voltage ratio of signal-to-noise. For the SN2 prototype probe, the resultant ultrasonic images from the data are presented in Figure 5.4. The results from the water tests showed improved performance of the SN2 probe to both detect and characterize (resolve) the spacing between the two pins.

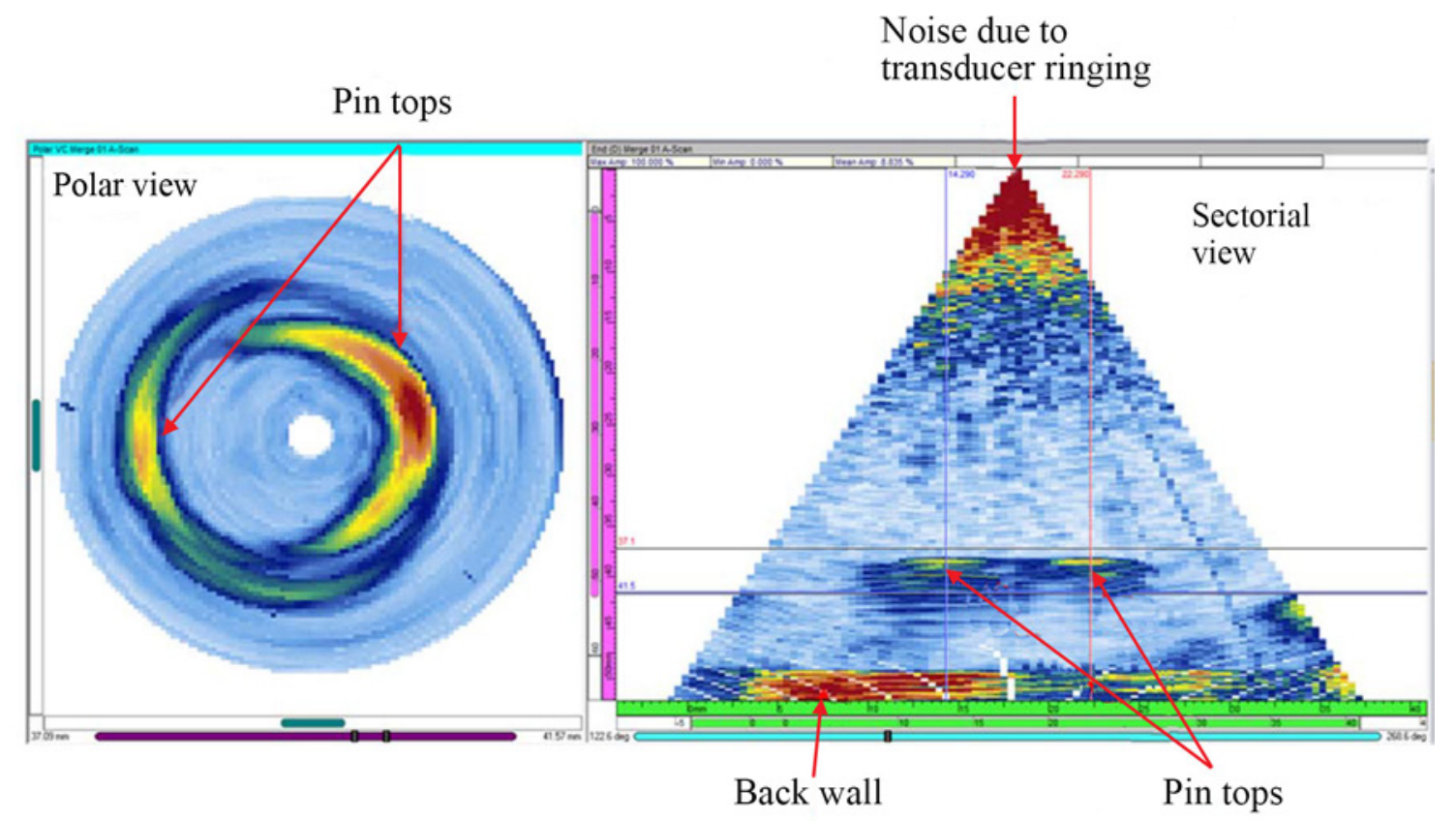

Figure 5.4. Ultrasonic Images Resulting from Scans of Position 1, Target 1, at 50-mm Standoff Height in Water for the SN2 Prototype Probe

Once again, the tops of both vertically oriented pins can be readily detected from the back wall response in water. The measured height of the pins was $11.3 \mathrm{~mm}$ and the separation between the pins was measured as $1.0 \mathrm{~mm}$. The SNR was $21 \mathrm{~dB}$, which corresponds to a factor of 11.2:1 in voltage ratio, of signal-to-noise. 



\subsection{Imaging Assessment in Sodium for Prototype Probes}

This section describes the sodium wetting challenges, the solution to mitigate those issues, and imaging tests and data obtained on the SN2 prototype probe in sodium, to compare and contrast with results from FY 2013 SN1 target detection trials in-sodium. The scanning configuration for the insodium tests is identical to that described in Section 5.0.

\subsection{SN2 Probe Face and Sodium Preparations Prior to in-Sodium Scanning}

This subsection describes the issues of transducer wetting, toward achieving effective transmission of ultrasonic energy through the face-plate of the PA-UT probe, and into the sodium for imaging purposes. Over the past few years, sodium wetting of the probe has become a challenging issue to overcome. In FY 2014, PNNL developed a process to consistently mitigate wetting problems. During the first test of the SN2 probe in-sodium in July 2014, no ultrasonic energy was transmitted into the sodium. Effective wetting was not being accomplished.

The sodium wetting issue is a combination of two primary factors in-sodium. The first is associated with the polish and surface finish of the probe face plate. While from a visual standpoint the face plate may appear suitably polished for optimal wetting, there is no proof that the wetting is effective from an ultrasonic standpoint. In FY 2010 through FY 2013, the differences in surface preparation of the various probes were noteworthy. It must be understood that the maximum displacement for a PZT-5A element is only about $350 \times 10^{-12} \mathrm{~m} / \mathrm{v}$. This simply means that the displacement between the surface of the $\mathrm{Ni}$ faceplate and the sodium is only a few microns, and any thin layer of contamination can preclude the energy from passing through.

The second wetting issue is associated with time in sodium and, more importantly, contaminants in the sodium; in particular, the oxide levels. There is nothing in the literature denoting a necessary time duration required at $260^{\circ} \mathrm{C}$ for a polished oxide-free surface, only information for unpolished surfaces full of oxide, so no clear guidance was available to the Technical Team on this matter. Our research indicated that clearly wetting was not an off-on condition; that a surface does not go from non-wetted immediately to full-wetted, but that wetting is a progressive phenomenon, described like a wave-front. It is a timetemperature dependent operation, and is also dependent on the oxide thickness, in which wetting starts, progresses, and then eventually becomes fully wetted over time. Effective wetting no doubt requires longer time on an unpolished oxide surface, and subsequently occurs more quickly on a polished surface. The Technical Team established a rule allowing at least 30 minutes at $260^{\circ} \mathrm{C}$ for wetting to appear, and for a stable signal response to be achieved. In addition, the literature mentions that for $\mathrm{Ni}$, the monooxide of $\mathrm{Ni}(\mathrm{NiO})$ is the usually expected species and is expected to dissolve, but that at high solubility levels of $\mathrm{O}_{2}$ in sodium, the ternary oxides of $\mathrm{Ni}$ have been seen, $\mathrm{NaNiO}_{2}$ and $\mathrm{Na}_{2} \mathrm{NiO}_{2}$. It says some ternary oxides can be more stable, and particularly more stable when high $\mathrm{O}_{2}$ levels are present. It does say that the $\mathrm{Ni}$ ternary oxides are expected to dissolve, but this still may be $\mathrm{O}_{2}$-level-dependent (Addison 1984). From trials performed at PNNL in FY 2013 and FY 2014, it has been shown that excessive levels of $\mathrm{O}_{2}(>3 \mathrm{ppm})$ are sufficient to preclude effective wetting on the PA-UT probe faces. 
In order to address these issues, the Technical Team developed a process for effective surface preparation and polishing of the Ni face-plate of the probe, prior to in-sodium testing. This surface treatment was done with a desk-mounted rotary polisher, with a 6-micron diamond lap, for the larger scratches, then employing a 1-micron diamond lap for a rough polish, all conducted in kerosene distillate oil and subsequently finished with a 0.05 sub-colloidal final polish, and rinsed well in ethanol and dried. A technician, manually holding the transducer, then spends approximately 10 minutes applying the 6-micron diamond lap initially because of the scratches on the face plate. Next, the technician spends approximately 5 minutes applying the 1-micron diamond lap, to further polish the face plate. Then, approximately 5 additional minutes are spent on the face plate, using a colloidal silica polish. The technician estimated this treatment regimen removed approximately $0.002-0.003 \mathrm{in}$. of material and that a lengthier treatment could be applied to remove $0.005-0.006$ in. of material, if necessary. A polishing cloth was used in the glove box for the final in-glove box polish, just prior to immersing the probe in sodium. It should be noted that this polishing/surface cleaning treatment must be repeated after exposure to air, not just Argon within the glove box. Exposure to air will initiate a rebuild-up of the surface oxides.

During the first SN2 prototype tests in July 2014, the test revealed a wetting issue in the sodium because of impurities in the argon glove box that precluded the introduction of acoustic energy into the sodium. The regeneration timer on the purifier was not working, which caused the levels of $\mathrm{O}_{2}$ to increase from less than $1 \mathrm{ppm}\left(\mathrm{O}_{2}\right.$ level of previous test with the $\mathrm{SN} 1$ probe $)$ to approximately $390 \mathrm{ppm}$. These $\mathrm{O}_{2}$ levels saturated the sodium and prevented the PA sensor from wetting in the sodium. In addition, the sodium batch used for these tests was the same batch used for FY 2013 testing; therefore, because of sodium use and age, other impurities may also have contributed to the failed test. During the spring and early summer of 2014, there were several months of glove box issues related to incomplete regeneration of glove box purifier beds from a broken regeneration timer, resulting in higher than normal $\mathrm{O}_{2}$ levels in the box atmosphere. The glove box purifier regeneration timer was subsequently replaced, allowing the box purifier to successfully complete a full regeneration cycle. So the purifier beds began to function and operate correctly. The freshly exposed sodium surface now indicated a suitable box atmosphere. The high $\mathrm{O}_{2}$ levels in the glove box during this period also saturated and destroyed the $\mathrm{O}_{2}$ sensor cell. The $\mathrm{O}_{2}$ sensor cell was also replaced during this time period. The glove box sensor compartment with the new $\mathrm{O}_{2}$ sensor cell was purged of air and the $\mathrm{O}_{2}$ levels were monitored. These levels slowly reduced to expected levels. New sodium was also ordered, received, and implemented in the containment. Finally, the glove box was brought to a fully functional state, the $\mathrm{O}_{2}$ sensor was consistently indicating correct $\mathrm{O}_{2}$ levels, and a fresh batch of sodium was prepared for the $\mathrm{SN} 2$ in-sodium target detection and imaging tests. Using these procedures, coupled with the surface preparation and polishing protocol, the SN2 probe successfully wetted in sodium.

\subsection{In-Sodium Testing Results}

Once again, for in-sodium scanning, the probes were unable to acquire data at the designed focal depth of $76.2 \mathrm{~mm}$, because of the constraints described in Section 5.0. These same limitations were present during the data acquisition tests for the SN1 prototype probe in FY 2013, so both sets of tests were conducted using identical scanning mechanisms, parameters, and sodium containment configurations. The ultrasonic images generated from in-sodium PA-UT scans and provided here were created using the Ultravision software, without the use of any image processing algorithm and were acquired at $260^{\circ} \mathrm{C}$. In this subsection, we again discuss the results from both prototype probes being 
scanned over position 1 of target 1 (dual pin set) where the data were again obtained using a 50-mm standoff between the probe and the base of the target. Figure 6.1 illustrates the imaging results from data acquired using the SN1 probe.

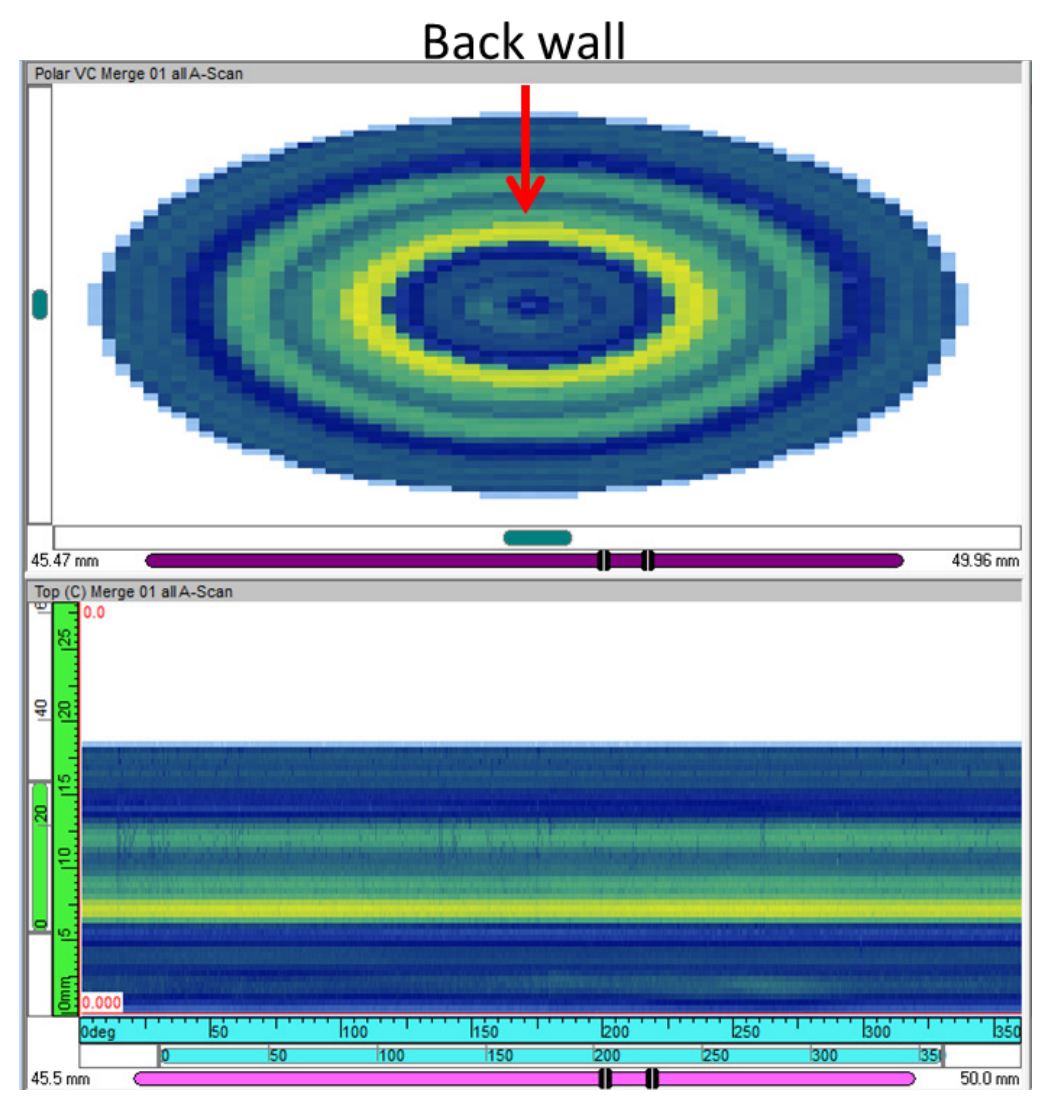

Figure 6.1. Ultrasonic Images Resulting from Scans of Position 1, Target 1, at 50-mm Standoff Height in Sodium, at $260^{\circ} \mathrm{C}$, for the SN1 Prototype Probe. Top: Polar view. Bottom: End view.

From a review of the SN1 data (obtained in FY 2013), only the back wall signal response is detected in the data. The top of the two vertical pins are not detected, and the anticipated dropout or "shadowing" of the ultrasonic field from the pins is not detected. The SNR for the back wall signal is $11 \mathrm{~dB}$, which corresponds to a factor of 3.55:1 in voltage ratio of signal-to-noise.

For the SN2 prototype probe, a sampling of the resultant ultrasonic images from the in-sodium data is presented in Figure 6.2. The results from the in-sodium tests showed some marginal improvement in detection performance and signal-to-noise for the SN2 probe to detect the signal response dropout (shadowing of the sound field) where the two vertical pins are located. 


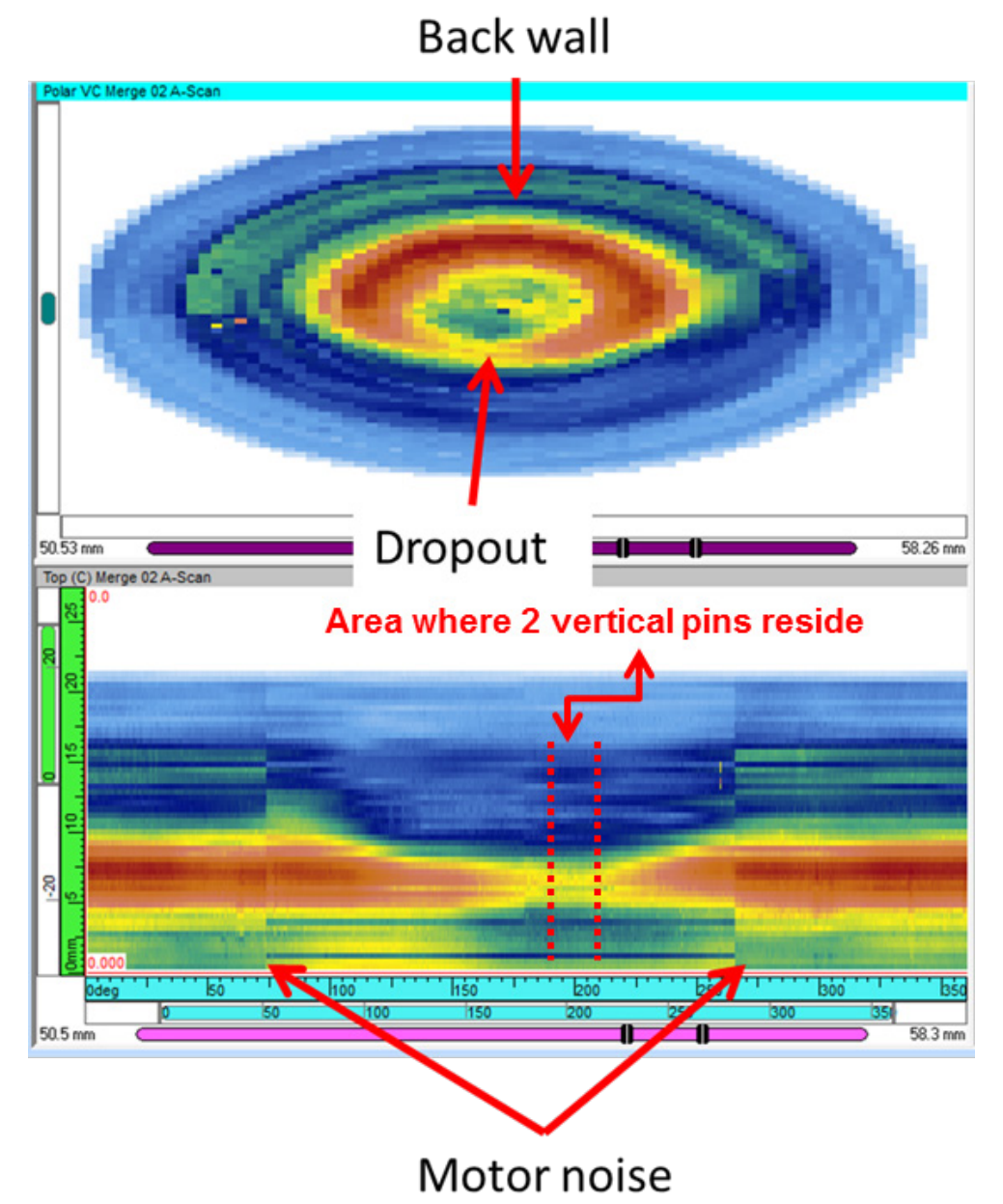

Figure 6.2. Ultrasonic Images Resulting from Scans of Position 1, Target 1, at 50-mm Standoff Height in Sodium, at $260^{\circ} \mathrm{C}$, for the SN2 Prototype Probe. Top: Polar view. Bottom: End view.

From a review of the SN2 data (obtained in FY 2014), the back wall signal response is detected more clearly in the data with a higher SNR. While the top of the two vertical pins are not detected, the anticipated dropout or "shadowing" of the ultrasonic field from the pins is detected. The SNR for the back wall signal is $17 \mathrm{~dB}$, which corresponds to a factor of 7.08:1 in voltage ratio of signal-to-noise. This reflects an increase in SNR by $6 \mathrm{~dB}$, or a factor of two of that of the SN1 probe. 


\subsection{Discussion and Conclusions}

This TLR provides an evaluation of data from both SN1 and SN2 prototype probes, obtained in FY 2013 and FY 2014, respectively, to establish a foundation for comparing and contrasting the imaging and detection performance of these probes in-sodium. This assessment includes target-detection/imaging performance of the probes in water and in-sodium, and focuses on the performance characteristics and primary inspection parameters described in Section 3.0. The results discussed here can be directly correlated to the measured performance characteristics of both probes reported previously (Diaz et al. 2014).

With regard to inspection time (duration), sodium temperature, and thermal cycling, both probes illustrated the required level of robustness to withstand any negative effects that could potentially impact probe performance. Both the SN1 and SN2 probes were tested in water after the in-sodium target detection trials were completed, and both probes demonstrated normal functionality and operability, verifying that no damage was incurred during in-sodium testing from temperature, thermal cycling, or the duration of testing in the glove box. In addition, all the data obtained in both FY 2013 and FY 2014 were acquired using multiple scanning repetitions to verify repeatability of data, and a suitable sample frequency (digitization rate) of $50 \mathrm{MHz}$, with no averaging. The $50-\mathrm{MHz}$ sample rate corresponds to 25 digitized points per cycle at an inspection frequency of $2.0 \mathrm{MHz}$. Finally, the spatial scanning increment step size for both polar $\left(360^{\circ}\right.$ rotation) scan increments and azimuthal $\left(-20^{\circ}\right.$ to $+20^{\circ}$ inspection angle) scan increments were sampled at $0.5^{\circ}$ steps in both FY 2013 and FY 2014. This allowed for a direct comparative analysis of the resultant data and images obtained from both SN1 and SN2 probes on the same targets in both water (at room temperature) and sodium (at $260^{\circ} \mathrm{C}$ ) environments.

While both SN1 and SN2 probes were designed to provide optimal focusing properties over the range of $76.2 \mathrm{~mm}$ to approximately $100 \mathrm{~mm}$, it was unfortunate that neither probe could be tested in-sodium at the proper focal distance. This was because of physical constraints within the glove box and with the scanning mechanism. Therefore, the assessment was conducted at $25-\mathrm{mm}$ and $50-\mathrm{mm}$ focal distances for both probes. While not optimal, the sound field dimensions and nominal operating frequencies of these probes were also different enough that one could indeed quantify marginal improvements in detection and imaging performance between the SN1 and SN2 probes, based on imaging results, SNR, detection and sizing capabilities in water, and to a lesser degree in-sodium. In addition to physical constraints, the motor noise issues (identified during data acquisition and illustrated in Figure 6.2) were periodically prevalent in the data as well, and this "system" effect is one that will be eliminated in FY 2015.

One of the key challenges that the Technical Team was able to overcome in FY 2014 was that of the sodium wetting of the probe's Ni faceplate, to enable effective transmission of ultrasonic energy into the sodium. This issue was discussed in subsection 6.1, but again, because of minor physical differences in the SN1 faceplate (concavity and thickness issues) to that of the SN2 faceplate, the Team was able to attribute a marginal improvement in overall performance to the SN2 probe, based on improved wetting in-sodium.

From a review of the data obtained in water, it is clear that the SN2 probe showed an improved ability to detect, resolve, and characterize the two vertical pins of Target 1, position 1. Sizing of the pin spacing was more accurate and the SNR was slightly improved. From a review of the sodium data, the metrics of 
SNR and signal dropout (shadowing) indicate an improved ability of the probe to detect the zone where the pins reside, and to capture a back wall signal with higher amplitude signal responses. These are critical steps in the evolution of a probe to eventually meet the detection and resolution criteria needed for a reliable and effective in-sodium phased-array examination probe and methodology.

The Technical Team assessed both probes using identical data acquisition and operational parameters. With the analyses of data obtained from these target-detection tests, the PNNL Technical Team was able to quantify marginal performance improvements between the two ETU probes, SN1 and SN2. In particular, incremental improvements in detection capability, resolution capability, SNR, and environmental robustness were identified. However, the Technical Team has concluded that the primary drivers (up to this point in the development process) inhibiting the achievement of suitable detection and resolution capabilities in-sodium, are:

- Inability to test the probes at the proper focal distance

- Reduced capability to steer and control a well-focused beam

- Inherent limitations of the 1D linear array design to provide suitable sound field characteristics and signal strength for achieving the required detection rates and resolution capability in-sodium.

Each of these limitations will be addressed by the PNNL Technical Team in FY 2015. While PNNL has demonstrated multiple 1D, phased-array probes that are robust, can effectively transmit ultrasonic energy in sodium, and can withstand the temperature and sodium environment, the primary FY 2015 challenge is to increase image resolution in-sodium. A 2D matrix array design will improve resolution and sensitivity, while increasing signal strength. In addition, this will provide an improved ability to control/steer the sound field, focus more energy with essentially the same aperture of the 1D design, and improve sound field focal dimensions. This FY 2015 effort will also develop an in-sodium PA-UT capability that will allow the acquisition of data in either rotational or raster-scan modes. Finally, the Technical Team will eliminate the constraints posed by the current scanning mechanism and sodium containment glove box, allowing for the evaluation and demonstration of this 2D matrix array probe at the optimal focal distance to the target. 


\subsection{References}

Addison CC. 1984. The Chemistry of the Liquid Alkali Metals. John Wiley \& Sons Ltd, New York.

Braatz BG, CL Aardahl, DL Baldwin, AD Cinson, JL Fernandes, AM Jones, PE Keller, MR Larche, R Mathews, CA Mullen, AF Pardini, GJ Posakony, ML Watkins, HT Chien, WP Lawrence, DM Engel and SH Sheen. 2013. FY2013 Joint Technical Report on Progress in Development of Ultrasonic UnderSodium Viewing Technology to Enable Inspection Systems for In-Service Inspection and Repair of Liquid Metal Fast Reactors. PNNL-22872, Pacific Northwest National Laboratory, Richland, Washington.

Diaz AA, DL Baldwin, AD Cinson, AM Jones, MR Larche, RA Mathews, CA Mullen, AF Pardini, GJ Posakony, MS Prowant, TS Hartman and MK Edwards. 2014. M3AR-14PN2301022 Technical Letter Report - Identify and Quantify the Mechanistic Sources of Sensor Performance Variation Between Individual Sensors SN1 and SN2. PNNL-23531, Pacific Northwest National Laboratory, Richland, Washington. 




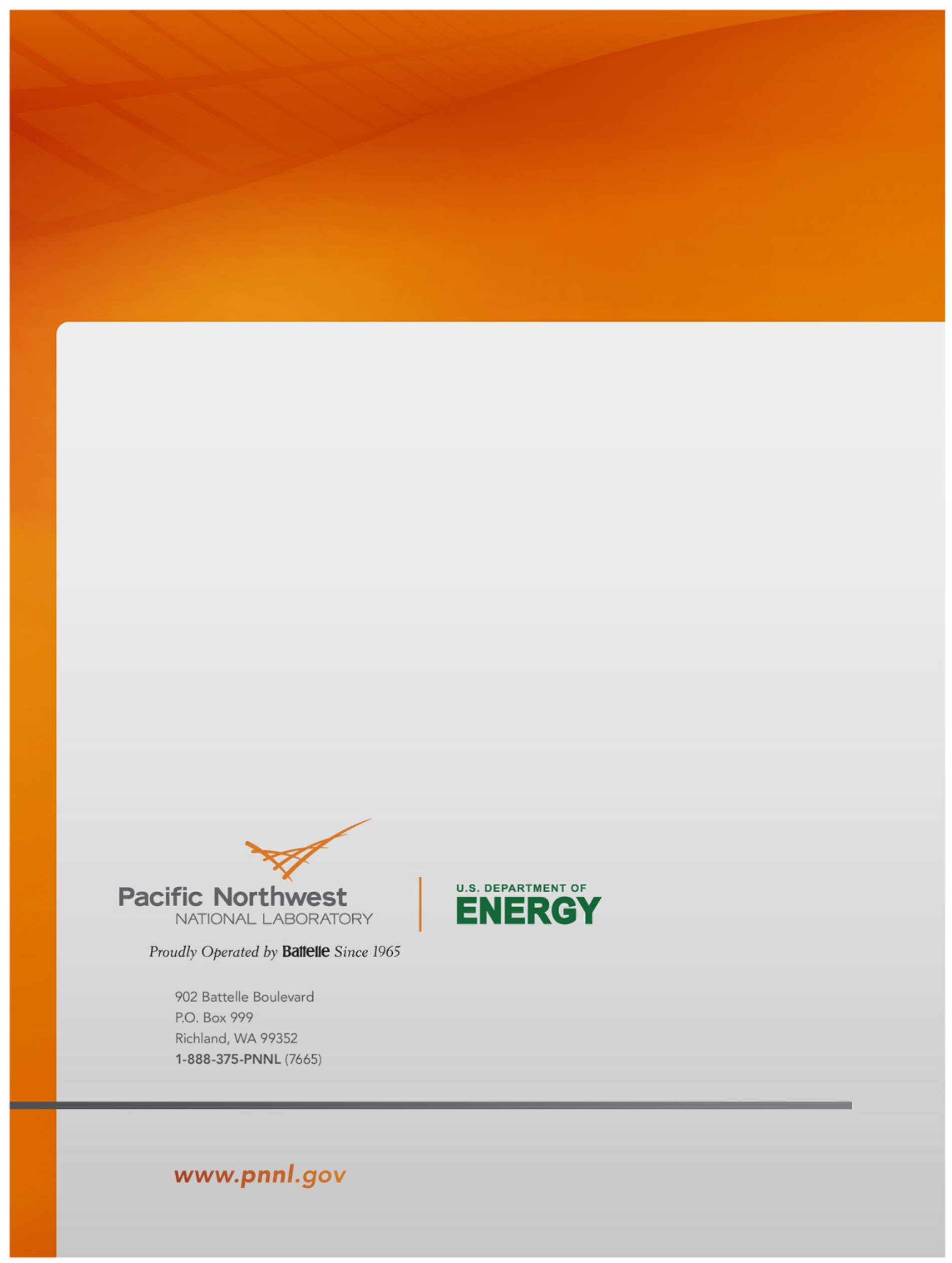

